\title{
On the evolution of simple shear in saturated soils
}

\author{
X. B. $\mathrm{Lu}^{1, *, \dagger}$, S. Y. Wang ${ }^{1}$ and Cui Peng ${ }^{2}$ \\ ${ }^{1}$ Institute of Mechanics, Chinese Academy of Sciences, 100080, Beijing, China \\ ${ }^{2}$ Institute of Mountain Hazard and Environment, Chinese Academy of Sciences, 610041, Chengdu, China
}

\begin{abstract}
SUMMARY
Development of shear bands in saturated soils is a multi-stage process based on the theoretical and numerical investigations in this paper. The soil is initially in homogenous shear strain state, and the instability can be characterized by a dimensionless number $D$. The inhomogenous distribution of shear strains appears when $D>1$, and the shear band will initiate and develop gradually. Numerical solutions show that only single shear band that is finally formed in the central region of the specimen even several disturbances (distributed along the specimen) appear in the beginning. Copyright (C) 2004 John Wiley \& Sons, Ltd.
\end{abstract}

KEY WORDS: simple shear; saturated soils; shear bands; instability

\section{INTRODUCTION}

Shear bands occur quite frequently in saturated soils under dynamic or static loading conditions and have attracted considerable attention for many years. Among the wealth of literature on this subject, we mention the classical works by Rice, Vardoulakis, Ottosen and Runesson et al. [1-6]. The necessary conditions for discontinuous bifurcations have been established by their works. Ottosen and Runesson et al. [3,6] presented the analytical results that include more general elastic-plastic models. The forming of the shear band in saturated soils may be described as follows. The strain or strain rate is significantly increased first, resulting in the build-up of high pore pressures and the decrease of the effective stresses. Under these conditions, the deformation of the saturated soils may behave very unusually. However, why may shear bands form in uniformly deformed saturated soils? How does the shear band evolve in saturated soils? All these questions are of great importance and interest in recent years [7-12]. Now it has been understood that the evolution of the shear bands in saturated soils is self-organized [8].

This paper aims to investigate the evolution of shear bands in saturated soils. Three stages are discussed respectively.

\footnotetext{
${ }^{*}$ Correspondence to: X. B. Lu, Institute of Mechanics, Chinese Academy of Sciences, 100080, Beijing, China.

${ }^{\dagger}$ E-mail: xblu@imech.ac.cn

Contract/grant sponsor: China National Natural Science Foundation (Key Yougth Foundation); contract/grant number: 40025103

Contract/grant sponsor: China National Natural Science Foundation; contract/grant number: 10202024

Received 22 November 2002

Revised 18 August 2003

Copyright (C) 2004 John Wiley \& Sons, Ltd. Accepted 30 October 2003
} 


\section{GOVERNING EQUATIONS AND ANALYSIS}

Although some criteria of instability have been obtained, these criteria cannot describe the temporal and special structure of the shear deformation i.e. the evolution. Therefore, a onedimensional model of simple shear of saturated soils is presented as follows for studying the evolution of shear bands [8]

$$
\begin{gathered}
\rho \frac{\partial^{2} \gamma}{\partial t^{2}}=\frac{\partial^{2} \tau}{\partial y^{2}} \\
C_{1} E_{r} \tau \dot{\gamma}=\frac{\partial p}{\partial t}-\frac{E_{r}}{K} \frac{\partial^{2} p}{\partial y^{2}}
\end{gathered}
$$

in which $p$ is the pore pressure, $E_{r}$ is the unloading modulus, $K=\rho_{\mathrm{w}} g / k, \rho_{\mathrm{w}}$ is the density of water and $k$ is the Darcy permeability, $g$ is the Earth gravity acceleration, $C_{1}$ is a material parameter, $\rho$ is the density of saturated soils mixture.

The complexity of the equations may be twofold. Firstly, the second equation in (1) is of the reaction-diffusion type, with the non-linear coupling source term $C_{1} E_{r} \tau \dot{\gamma}$. The first equation in (1) may lose its hyperbolic properties at the peak of the stress-strain path $f(x)$. Because of the vanishing wave speed $c=\sqrt{\dot{f}(x) / \rho}$, this may cause a strong discontinuity near the loading boundary which may be one of the possible mechanisms about the concerned shear location. Secondly, scaling the governing equations properly is of crucial significance in the analysis of the shear band in saturated soils, because different scales of time and size involved in this phenomenon. Obviously the half width of the band is much less than the size of the specimen $L$. More importantly, there are several time scales, such as $1 / \dot{\gamma}^{*}, L /\left(\tau^{*} / \rho\right)^{0.5}, L \mu / \rho_{\mathrm{w}} g k$, etc. characterizing effects of rate, wave, pore pressure transfer etc. Most of all, maybe the two time scales $t_{\mathrm{d}}=\rho \gamma^{*} \delta^{*^{2}} / \tau^{*}, t_{\mathrm{h}}=\delta^{*^{2}} \rho_{\mathrm{w}} g / k p^{*}$, representing rate-dependent diffusion $\tau^{*} / \rho \gamma^{*}$ and pore pressure diffusion $k$, are of most importance in the development of shear bands in saturated soils ( $\rho_{\mathrm{w}}$ is the density of water). The dimensionless ratio $t_{\mathrm{h}} / t_{\mathrm{d}}=\rho_{\mathrm{w}} g \tau^{*} / k p^{*} \rho_{\mathrm{s}} \gamma^{*}$ defines a number $C_{r}$, which is a key parameter in the following discussion.

If the appropriate time and space scales under discussion are denoted by $t_{k}$ and $y_{k}$ as follows: the dimensionless governing equations should be $[8,13]$

$$
\begin{gathered}
a \frac{\partial \dot{\bar{\gamma}}}{\partial \bar{t}}=\frac{\partial^{2} \tau}{\partial \bar{y}^{2}} \\
\bar{\tau} \dot{\bar{\gamma}}=b \frac{\partial \bar{p}}{\partial \bar{t}}-d \frac{\partial^{2} \bar{p}}{\partial \bar{y}^{2}}
\end{gathered}
$$

in which $a=\rho \gamma^{*} y^{*^{2}} / t^{*^{2}} \tau^{*}, b=p^{*} / \tau^{*} \gamma^{*} t^{*}, d=E_{r} p^{*} / K y^{*^{2}} \tau^{*} \gamma^{*}$, all variables with bar '-' are dimensionless.

There are three representative choices of $t_{k}$ and $y_{k}$ to scale the above equations (Table I).

Case 1 represents the early stage $\left(t \cong t_{\mathrm{d}}\right)$ of localization, which can be described by isobar (equal super-pore-pressure everywhere) and moment dominated model. Case $2\left(t \cong t_{\mathrm{h}}\right)$ represents that the localization shear may be formulated by quasi-static approximation, which is dominated by pore pressure production and diffusion. If one intends to investigate the phenomenon in the range of time $t_{\mathrm{h}}$, but pays attention to the gauge length scale $l>y_{k 3}=$ $\sqrt{p^{*} / \rho \gamma^{* 2}}$, the resulting simplification would be no pore water exchanging at the boundary of 
Table I. Three representative choices of $t_{k}$ and $y_{k}$ to scale $a, b, d$.

\begin{tabular}{cccccc}
\hline & $t_{k}$ & $y_{k}^{2}$ & $a$ & $b$ & $d$ \\
\hline 1 & $\rho E_{r} p^{*} / K \tau^{*}$ & $E_{r} p^{*} / K \tau^{*} \dot{\gamma}^{*}$ & 1 & $C_{r}$ & 1 \\
2 & $p^{*} / C_{1} E_{r} \tau^{*} \dot{\gamma}^{*}$ & $E_{r} p^{*} / K \tau^{*} \dot{\gamma}^{*}$ & $1 / C_{r}$ & 1 & 1 \\
3 & $p^{*} / C_{1} E_{r} \tau^{*} \dot{\gamma}^{*}$ & $p^{*} / \rho \dot{\gamma}^{*}$ & 1 & 1 & $1 / C_{r}$ \\
\hline
\end{tabular}

band, as shown in case 3. Therefore, no pore water exchanging approximation is a look-over from outside of the band itself. The approximation may be leading, when the evolution of the shear band is the subject of investigation.

Above all, cases 1 and 2 are the two most important approximations, depicting the early and late stages of the shear localization. The common point in the two stages is that both are dissipative, although the physics is different, i.e. rate-dependence and pore pressure diffusion respectively.

\section{INSTABILITY AND LOCALIZATION}

The occurrence of instability is presumed to be responsible for the initiation of the shear bands. In the linear analysis [14-19], the condition for instability and the growth rate of infinitesimal nonuniformities were obtained. In particular, the analysis shows that a dimensionless parameter D plays a more significant role than that significant pore pressure transfer takes place at the moment of instability, where

$D=\frac{(\partial \tau / \partial p) \tau}{k(\partial \tau / \partial \gamma)}=\frac{(\partial \tau / \partial p) \mathrm{d} p \tau \mathrm{d} \gamma}{k \mathrm{~d} p(\partial \tau / \partial \gamma) \mathrm{d} \gamma}=\frac{\text { plastic work due to } \mathrm{d} \gamma}{\text { pore pressure increase } \mathrm{d} p} \cdot \frac{\text { pore pressure softening due to } \mathrm{d} p}{\text { stain hardening due to } \mathrm{d} \gamma}$

According to the analysis, the assumption of no pore water exchanging at the boundary of band is an appropriate approximation for instability study. In this case, $D>1$ manifests the occurrence of instability. Another point worthy mentioning is that inertial is the factor, being responsible for the non-linearity of the characteristic equation in no pore water exchanging condition [9].

\section{SHEAR BANDS}

Although some analyses about the instability and localization provide some operational rules to signify the occurrence of shear bands, all these approaches do not reveal the evolution process of shear bands. Indeed, this is the most difficult issue of the problem owing to its non-linearity and transient performance.

Richar et al. [20] and Khali et al. [21] reported multi-stages of shear localization in water-saturated soils, localization does not happen simultaneously and homogenously on the circumference of specimen. All observations imply that shear banding appears to 
be a series of processes from instability to fully developed shear band, and finally leading to fracture.

In fact, according to the above-mentioned scaling law, quasi-static pore pressure diffusion dominated approximation is a suitable model to describe the process. The plastic work rate, acting as the source of super pore pressure, is the unique non-linear term in the coupling equations. For simplicity, a linear rate and pore pressure dependent constitutive relation is assumed as $\tau / \tau^{*}=1+\gamma / \gamma^{*}-p / p^{*}$. Therefore, the uniform but unsteady stress is $\tau(t, y)=$ $\tau(t)=\tau^{*}\left(1-\left(p_{\delta}(t) / p^{*}\right)\right)$. Compared to the local shear, the material outside is regarded as rigid. Then the boundary velocity could be expressed by means of the variables within the localized zone as [8]

$$
v=\int_{0}^{\delta} \dot{\gamma} \mathrm{d} y=\dot{\gamma}^{*} \int_{0}^{\delta}\left(\frac{p}{p^{*}}+\frac{\tau}{\tau^{*}}-1\right) \mathrm{d} y=\dot{\gamma}^{*}\left(\int_{0}^{\delta(t)} p(t, y) \mathrm{d} y-p_{\delta} \delta(t)\right) / p^{*}
$$

where $\delta$ is the half width of shear band, $p_{\delta}$ is the pore pressure at boundary, $*$ denotes the characteristic parameter.

By differentiating the equation above, one may obtain the following differential equation of the band evolution:

$$
\frac{\mathrm{d} \delta}{\mathrm{d} t}=\left\{\bar{\tau} \overline{\dot{\gamma}}-\left(\left.\frac{\partial^{2} p}{\partial y^{2}}\right|_{\delta}-\frac{\bar{\partial}^{2} \bar{p}}{\partial y^{2}}\right)\right\} /\left(\frac{\partial p}{\partial y}\right)
$$

Because of the pore pressure gradient $\partial p /\left.\partial y\right|_{\delta}<0$, plastic work rate $\bar{\tau} \overline{\dot{\gamma}}>0$ drives the deformed region narrow, while pore pressure diffusion $\partial^{2} p /\left.\partial y^{2}\right|_{\delta}-\bar{\partial}^{2} \bar{p} / \partial y^{2}>0$ makes the region wide. Obviously, the band-like shear zone is self-organized by means of the two opposite mechanisms. Interestingly, in the analysis the non-linearity of the problem has been minimized to only one term-the plastic work rate [8].

Although the above model sheded light on the mechanism of shear band in saturated soils, it is extremely oversimplified, so cannot be realistic. In order to investigate the process in detail, some numerical simulations and experiments have been done [11,22]. Careful examinations of the same specimen revealed several fine shear bands distributing over the deformed region (Figure 1). On contrary, only one fully developed shear band appeared when fracture initiated.

Based on Equation (1), numerical simulation is carried out in order to reveal how disturbances of finite amplitudes evolve into shear band. Figures 2, 3 show that the dimensionless stress versus dimensionless time $t$. The line represents disturbed stress with finite amplitude of one and three disturbances, which are added in the vicinity of stress climax. Figures 5, 7 show the variation of disturbed strain rate, from which we can see that drastic increase of shear strain rate occurring at about $t=0.1 \sim 0.15 \mathrm{~s}$. It is clear that the stress retains close to the uniform solution when $t$ is less than $0.1 \sim 0.15 \mathrm{~s}$ and then declines gradually and shows apparent stress drop (Figures 4,6 ). To simulate a number of fine shear bands appearing near stress peak in experiments, one and three disturbances (distributed along the specimen) are adopted in the computation. All cases show that similar late-stage behaviour, namely single shear band appearing in the central region. Here, the three disturbances gradually merge into a contracting localized area at about $t=0.1 \sim 0.15 \mathrm{~s}$, where the stress begins to decline. Afterwards, as the strain rate and pore pressure of the shear band increase rapidly, the band shrinks. Finally, the shear band is fully formed and shown quite stable bandwidth. All these figures are qualitatively consistent with observations and are in fairly good agreement with the analytical prediction [6]. Nevertheless, it is not obvious that the softening branch becomes 


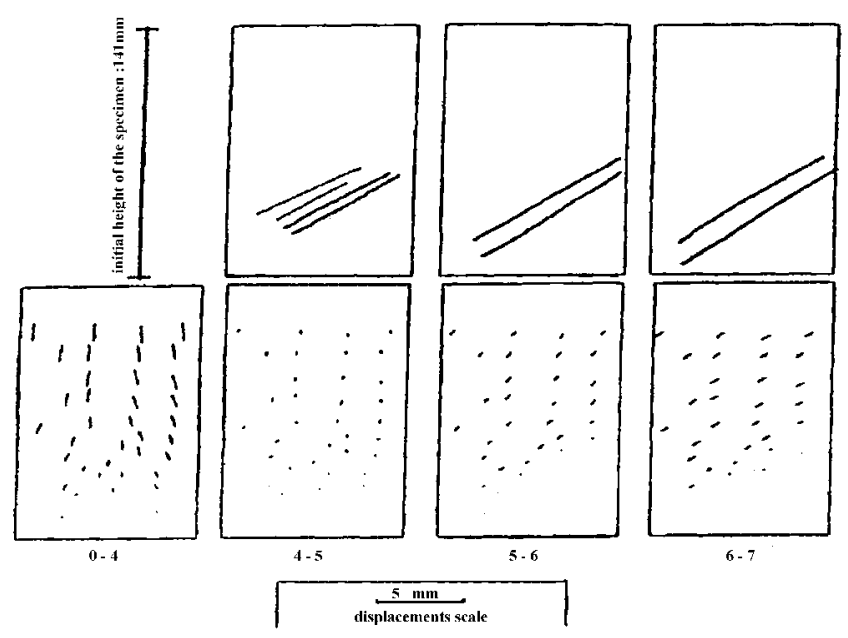

Figure 1. X-ray images of shear bands.

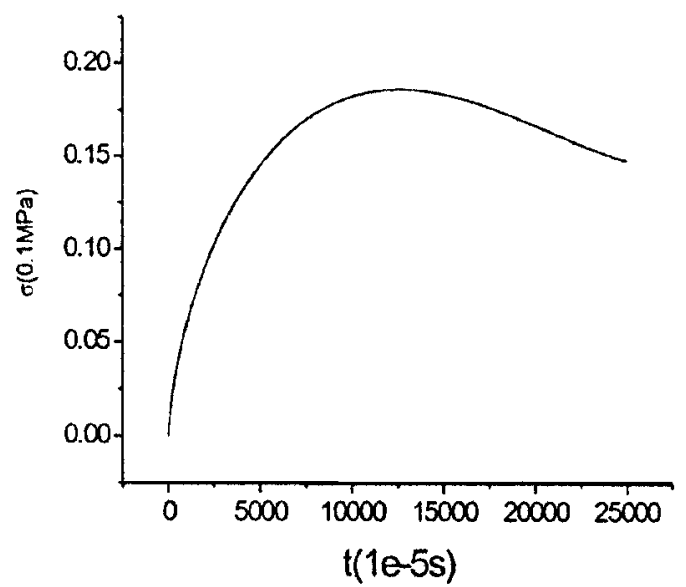

Figure 2. The evolution of the stress vs time under three disturbances condition.

steeper and the shear band becomes thinner. This may be of the absence of a characteristic length in our model. By introducing a characteristic length e.g. strain gradient, we will see a obvious evolution of a steeper softening branch and a thinner band [11].

In the numerical simulations, the constitutive relation adopted is as follows:

$$
\tau=a_{1} \gamma^{l} \dot{\gamma}^{m} p^{n}
$$

in which $a_{1}, l, m, n$ are constant coefficients.

The parameters are adopted as follows:

$$
\begin{aligned}
& k=9 \times 10^{7} \mathrm{~m}^{2}, \rho=2400 \mathrm{~kg} / \mathrm{m}^{3}, E_{r}=4 \times 10^{7} \mathrm{~m}^{2} \\
& a_{1}=5000, l=1, m=1, n=-0.25 .
\end{aligned}
$$




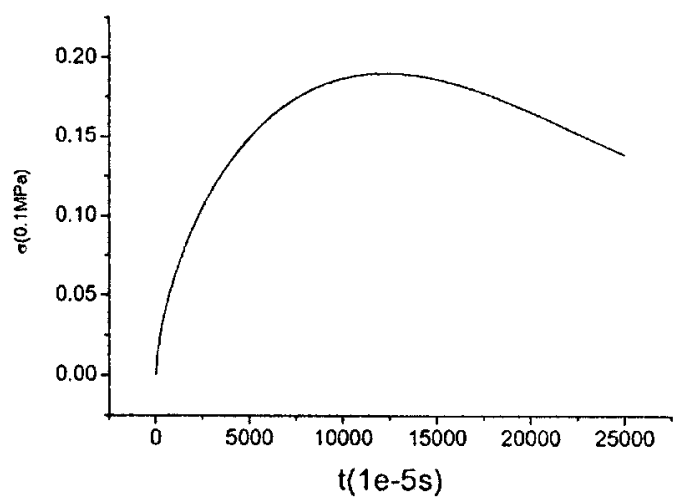

Figure 3. The evolution of the stress rate vs time under three disturbances condition.

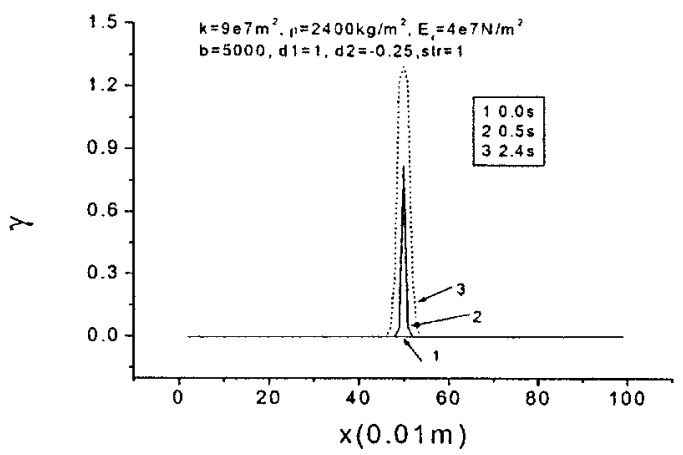

Figure 4. The evolution of the strain vs length under one disturbance condition.

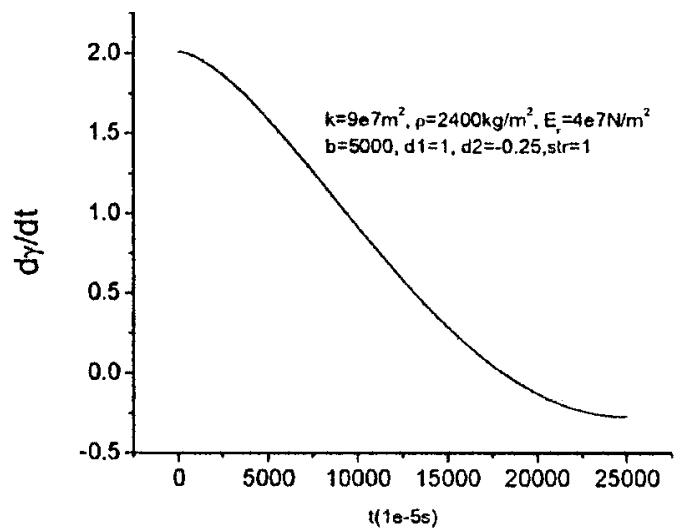

Figure 5. The evolution of the strain rate vs length under one disturbance condition. 


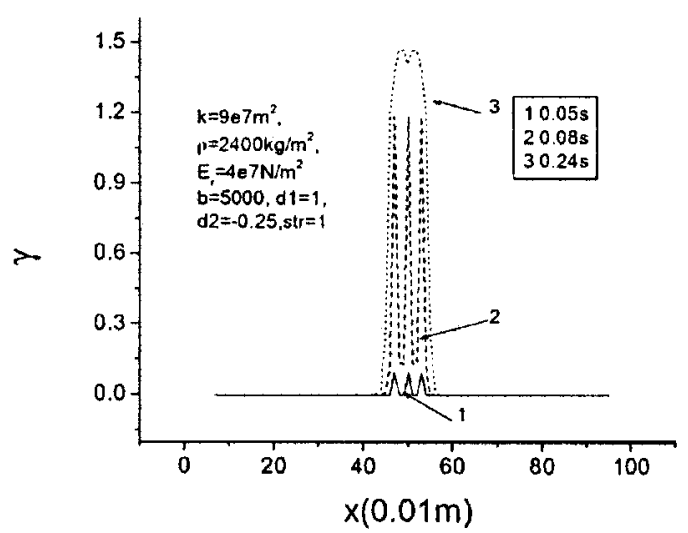

Figure 6. The evolution of the strain vs length under three disturbances condition.

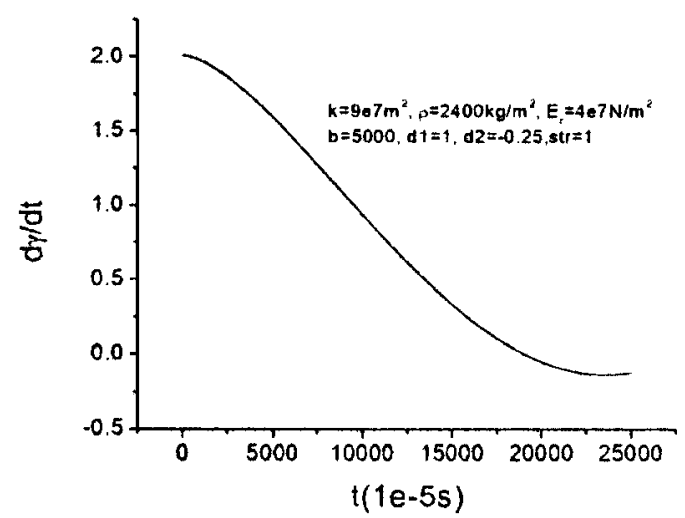

Figure 7. The evolution of the strain rate vs time under three disturbances condition.

The initial and boundary conditions:

$p(0, y)=0.39 \mathrm{MPa}, \gamma(0, y)=0$

$\dot{\gamma}=2.0$, except that the values are $1 \%$ higher at the points $0.48 L, 0.5 L, 0.52 L, L$ is the length of specimen.

\section{QUASI-STEADY LATE-STAGE BEHAVIOUR}

In accord with the scaling law of the governing equations, if we examine the local area $y_{k}^{2}=$ $E_{r} p^{*} / K \tau^{*} \dot{\gamma}^{*}$ at longer time $t_{k}=N p^{*} / C_{1} E_{r} \tau^{*} \gamma^{*}$ with $N \gg 1$, the three dimensionless parameters would become $a \approx 1 / N \cdot C_{r}, b=1 / N, d=1$. The concerned problem becomes not only quasistatic, but also quasi-steady in pore pressure. Since the observed shear bands should be its late 
stage appearance, this asymptotic steady state would be very helpful to understand the structure of the shear band in saturated soils [3]. It has been pointed that this quasi-steady solution can be found in general rate and pore pressure dependent constitutive relation $\dot{\gamma}=f(\tau, p)$, by integrating the second equation of (1) we may obtain

$$
y=\sqrt{\overline{2 \tau(t)}} \int_{p}^{p_{m}(t)}\left(1 / \int_{\xi}^{p_{m}(t)} f(\tau(t), \eta) \mathrm{d} \eta\right)^{0.5} \mathrm{~d} \xi
$$

in which $p_{m}$ is the pore pressure at the centre of the shear band. Let $p=P_{\delta}$, the boundary pore pressure of the shear band, the above expression gives the half width of the band.

The significance of the quasi-steady late stage solution can be verified from another reasoning. If we skip over the transient process of the pore pressure assisted shear and pay attention to the late stage state only, the linearized operator of the equations would give enough information about the bifurcation of steady solutions, i.e. the exchange of stabilities. In the case of rate and pore pressure dependent constitutive relation, the condition of pore pressure softening outweighing strain rate hardening signifies the transition from node to saddle, namely loss of stability of uniform shear deformation. Then the new steady solution would be asymptotically stable, hence represent the late stage behaviour of the shear deformation.

$$
\left(-\frac{\partial \tau}{\partial p}\right) \tau=\lambda\left(\frac{\partial \tau}{\partial \dot{\gamma}}\right) \beta^{2}
$$

in which $\beta$ is the wave number.

The late stage behaviour can be estimated by simply balancing the two terms of pore pressure source and diffusion in quasi-steady energy equation as [23]

$$
\delta \approx\left(E_{r} p^{*} / K n \tau^{*} \dot{\gamma}^{*}\right)^{0.5}
$$

\section{CONCLUSIONS}

The theoretical and numerical investigations show that the development of the shear band in saturated soils is a multi-stage process. The process may begin from homogenous shear strain state. Once the dimensionless number $D>1$, inhomogenous shear distribution appears and the shear band will form gradually. The numerical solutions show that even if there are some disturbances in the beginning, only one band forms at last.

\section{NOMENCLATURE}

$\begin{array}{ll}* & \text { within the band } \\ \tau & \text { shear stress } \\ \gamma & \text { shear strain } \\ p & \text { pore pressure } \\ \rho_{\mathrm{w}} & \text { density of water } \\ \rho & \text { density of grains } \\ L & \text { length of the specimen } \\ g & \text { the Earth gravity acceleration }\end{array}$




$\begin{array}{ll}\mu & \text { viscosity of water } \\ k & \text { Darcy permeability } \\ C_{1} & \text { material parameter } \\ E_{r} & \text { compressible modulus of grains } \\ t_{\mathrm{h}}, t_{\mathrm{d}} & \text { characteristic times } \\ \delta & \text { half width of shear band } \\ a, b, d & \text { dimensionless parameters } \\ C_{r} & \text { dimensionless characteristic time ratio } \\ a_{1}, l, & \text { constant coefficients } \\ m, n & \\ p_{\delta} & \text { pore pressure at boundary }\end{array}$

\section{ACKNOWLEDGEMENTS}

This programme is supported by the China National Natural Science Foundation-Key Yougth Foundation (No. 40025103) and China National Natural Science Foundation (No. 10202024).

\section{REFERENCES}

1. Rice JR. On the stability of dilatant hardening for saturated rock masses. Journal of Geophysical Research 1975; 80(11):1531-1536.

2. Vardoulakis I. Stability and bifurcation of undrained, plane rectilinear deformations on water-saturated granular soils. International Journal for Numerical and Analytical Methods in Geomechanics 1985; 9:399-414.

3. Ottosen NS, Runesson K. Properties of bifurcation solutions in elasto-plasticity. International Journal of Solids and Structures 1991; 27:401-421.

4. Runesson K, Ottosen NS, Peric D. Discontinuous bifurcations of elastic-plastic solutions at plane stress and plane strain. International Journal of Plasticity 1991; 7:99-121.

5. Runesson K, Peric D, Sture S. Effect of pore-fluid compressibility on localization in elastic-plastic porous solids under undrained conditions. International Journal of Solids and Structures 1996; 33(10):1501-1518.

6. Rice JR, Cleary MP. Some basic stress diffusion solutions for fluid-saturated elastic porous media with compressible contents. Reviews on Geophysics and Space Physics 14; 227-241.

7. Larsson R, Larsson J, Runesson K, Sture S. Localization in an undrained hyperelastoplastic porous medium. Poromechanics A Tribute to Maurice A. Biot. Thimus et al. (eds). Balkema: Rotterdam, 1998; 99-104.

8. Lu Xiaobing Zhang Jiahua, Yang Zhengsheng. On the evolution of shear bands in saturated soil. International Journal of Nonlinear-Mechanics 2000; 35:21-26.

9. Lu Xiaobing. On the shear instability of saturated soil. International Journal of Engineering Science 2001; 39: 963-972.

10. Vardoulakis I. Dynamic stability analysis of undrained simple shear on water-saturated soils. International Journal for Numerical and Analytical Methods in Geomechanics 1986; 10:177-190.

11. Lu XB. On the development of shear band by considering the strain gradient. Iranian Journal of Science and Technology 2003; 27(b1):57-62.

12. Loret B, Provest JH. Dynamics strain localization in fluid-saturated porous media. Journal of Engineering Mechanics (ASCE) 1991; 117:907-922.

13. Bai YL. Evolution of thermo-visco-plastic shearing. Institute of Physics Conference of Oxford 1989; 102(3):75-86.

14. Lade PV, Nelson RB, Ito YM. Instability of granular materials with nonassociated flow. Journal of Engineering Mechanics (ASCE) 1988; 114:2173-2191.

15. Lade PV. Instability and liquefaction of granular material. Computers and Geotechnics 1994; 16(2):123-151.

16. Vardoulakis I, Graf B. Calibraten of constitutive models for granular materials using data from biaxial experiments. Geotechnique 1985; 35:299-317.

17. Peric D, Runesson K, Sture S. Evaluation of plastic bifurcation for plane strain versus axisymmetry. Journal of Engineering Mechanics (ASCE) 1992; 118(3):512-524.

18. Pietruszczak S, Niu X. On the description of localized deformation. International Journal for Numerical and Analytical Methods in Geomechanics 1993; 17:791-805. 
19. Pietruszczak S. Undrained responses of granular soil involving localized deformation. Journal of Engineering Mechanics (ASCE) 1995; 121(12):1292-1297.

20. Richard JF, Wendell WH, Michael AM et al. Strain localization and undrained steady state of sand. Journal of Geotechnical and Geoenvironmental Engineering (ASCE) 1996; 122(6):462-473.

21. Alshibbi KA, Sture S. Shear band formation in plane strain experiments of sand. Journal of Geotechnical and Geoenvironmental Engineering (ASCE) 2000; 126(6):495-503.

22. Oda M, Kazama H. Microstructure of shear bands and its relation to the mechanisms of dilatancy and failure of dense granular soils. Geotechnique 1998; 48(4):465-481.

23. Prisco CDi, Imposimato S, Aifantis EC. A visco-plastic constitutive model for granular soils modified according to non-local and gradient approaches. International Journal for Numerical and Analytical Methods in Geomechanics 2002; 26:121-138. 